\title{
Innovating problem solving in power quality devices: a survey based on Dynamic Voltage Restorer case (DVR)
}

\begin{abstract}
The theory of inventive problem solving (TRIZ) is one of emerging creative problem solving methodologies. This paper proposes a way to simplify TRIZ for electrical domain usage, while making it feel less complex, more relevant and understand able for users in a specific sector. This research takes Dynamic Voltage Restorer (DVR) device as a case from electrical engineering domain, to demonstrate the proposed TRIZ based guidance framework development which will assist in solving problems of DVR devices. The proposed sector specific guidelines in particular segment of Electrical Engineering (e.g. power quality device DVR) will be closer, more comprehensible and particularly linked to key parameters of that sector. By using the derived guidance framework, field engineers with very basic TRIZ knowledge may apply TRIZ confidently for design and problem solving purpose.
\end{abstract}

Keyword: TRIZ; Complexity; Power quality; Guidance framework 